\title{
Highly Performing Nanocomposite Sensors for Damage Detection ${ }^{+}$
}

\author{
Erika Magnafico, Maryam Karimzadeh and Giulia Lanzara * \\ Engineering Department, University of Rome, RomaTre, 00146 Rome, Italy; \\ erikamagnafico92@gmail.com (E.M.); giulia.lanzara@uniroma3.it (M.K.) \\ * Correspondence: giulia.lanzara@uniroma3.it \\ + Presented at the 18th International Conference on Experimental Mechanics (ICEM18), Brussels, Belgium, \\ 1-5 July 2018.
}

Published: 20 June 2018

\begin{abstract}
Due to their superior physical and electro-mechanical properties, Carbon Nanotubes (CNTs) are one of the most promising composite fillers to realize ultralight and flexible strain sensors that can be used, among others, to monitor strain concentrations within a structure when damage occurs. In this study, sensors are made of Multi-walled Carbon Nanotubes (MWNTs) embedded in a Polymide (PI) matrix. Nanocomposites are characterized under no-load conditions to study the electrical properties, and under tensile loading conditions, to evaluate the electromechanical and piezoresistive response. The results highlight a two orders of magnitude decrease in electrical resistivity if compared with previous studies, the capability to instantaneously respond to unpredictable deformations and to easily adapt to three-dimensional shapes. The beauty of the as conceived nanocomposite film, if compared with the commercially available strain gages, is its unprecedented potential expandability to monitor larger areas without the loss of ultra-low local (in scale) detection. Local detection is in fact allowed by nanoscale morphology changes that induce changes in local electrical conduction. The selected polyimide matrix allows the use of the proposed sensor to harsh and high temperature environments while keeping high flexibility and excellent mechanical properties, key parameters for the realization of reliable electromechanical films.
\end{abstract}

Keywords: carbon nanotube; nanocomposites; strain sensing

\section{Introduction}

Carbon Nanotubes (CNTs), since they were discovered, have drawn a large attention for their unique chemical, thermal, mechanical and electro-mechanical properties that make them very interesting in several engineering fields. Recent studies are focused on the use of this material in strain sensing. It is known that, by embedding CNTs in an insulating polymer matrix, it is possible to realize conductive piezoresistive nanocomposites with a good capability to sense strain. These nanocomposites represent a good alternative for developing a new promising type of strain sensor.

When CNTs are dispersed in the polymer matrix, they form a conductive network through which the current flows. The resistivity of the material is directly related to the conductive network configuration at the nanoscale: if the material is strained, the gap between the neighboring carbon nanotubes increases, leading to an increase in terms of resistivity [1]. Park et al. reported an increase in electrical resistance of epoxy/multi-walled carbon nanotube films under tensile stress [2].

Several authors have compared the piezoresistive behavior of CNT nanocomposite films with the response of films made of bare carbon nanotubes for strain sensing applications. Kang et al. used in particular buckypapers and SWNTs-PMMA nanocomposites. It was observed that the buckypaper had a higher sensitivity wthin a smaller strain range. On the other hand, the composite showed a 
linear symmetric strain response during tension and compression conditions [3]. Li et al. investigated the piezoresistive behavior of thin MWNTs films. The material was characterized by uniaxial load/unload tensile tests [4]. Other factors, such as the sonication time and temperature can influence the electro-mechanical material response. Bu et al. studied the effect of sonication time on the sensitivity of CNTs strain sensor and found that a long sonication time improved considerably the sensor sensitivity [5].

Vemuru et al. also used carbon nanotubes as strain sensors. It was shown that there is a linear relationship between the voltage changes and the strain changes [6].

Conventional strain gages are broadly used to monitor strain in structures. But these devices require complex installation procedures and can't be used for distributed strain measurements. They can in fact measure strain only locally (where they are bonded) and along a specific direction in agreement with their serpentine design. Novel ultra-light sensing devices are necessary to overcome these limitations. Recent efforts by the authors.

In a previous study by the author, the electromechanical response of MWNTs-PMMA nanocomposites was investigated. Films with different amounts of CNTs were fabricated and electromechanically characterized in order to evaluate the resistivity range of the material. Best performance was found in films made with a 7\% MWNTs weight fraction. This result suggests that a further improvement in the electromechanical response could be reached by increasing the CNTs content dispersed in the polymeric matrix. However, the authors observed that with increasing CNTs content, the nanocomposite increased its fragility [7] and this is in conflict with the creation of mechanically flexible and reliable electromechanical films.

To overcome these limitations, the electromechanical films should be made of a polymeric matrix that is characterized by advanced mechanical properties, and, at the same time, a larger amount of CNTs should be dispersed in such matrix. In this way the electrical response, thus an overall electromechanical response, should be enhanced. With this in mind, the purpose of this experimental work, is to realize, characterize and test with an unconventional setup, a polyimide (PI)based sensing film integrated with a large amount of MWNTs. Polyimide in fact has excellent mechanical and thermal properties and is ideal for the scope of this study.

\section{Materials and Methods}

Multi-walled Carbon Nanotubes (MWNTs) from US Research Nanomaterials, Inc. are used (purity higher than 95\%, outer diameter between 50-80 nm, inner diameter between 5-15 $\mu \mathrm{m}$, net weight of $100 \mathrm{~g}$, length of the tube between 10-20 $\mu \mathrm{m}$ ). Polyamic acid (PAA), precursor of polymide (PI), is employed for the nanocomoposite films fabrication. The solvent casting technique is adopted.

The n-methyl-2 pyrrolidinone (NMP) solvent is used to disperse MWNTs, making a solution with $0.6 \%$ of MWNTs (weight fraction). In order to obtain a homogeneous MWNTs/NMP suspension, the mixture is ultrasonicated for $45 \mathrm{~min}$ at room temperature. During the sonication process, PAA is mixed with NMP on a magnetic stirring plate for $20 \mathrm{~min}$. Afterwards, the MWNT/NMP solution is added to the PAA/NMP suspension obtaining a mixture of MWNT/PAA made of $12 \mathrm{wt} \%$ of MWNTs, and $88 \mathrm{wt} \%$ of PAA. This obtained viscous solution is ultrasonicated again for $20 \mathrm{~min}$ at room temperature and then is poured onto a glass mold and dried in a vacuum oven at $95{ }^{\circ} \mathrm{C}$ for $1.5 \mathrm{~h}$ to let the solvent evaporate. The MWNT/PAA nanocomposite films are cured in an air circulating oven: a first curing at $200{ }^{\circ} \mathrm{C}$ for $30 \mathrm{~min}$ followed by a second curing at $350{ }^{\circ} \mathrm{C}$ for $1 \mathrm{~h}$. During this process, amide linkages convert into imide linkages thus delivering the MWNT/PI nanocomposite films.

\section{Results and Discussion}

To investigate the resistivity range of the material, electrical tests in stress-free condition are performed by using a Semiconductor Parameter Analyzer (Agilent). Electrical contacts are made with a conductive carbon paste and wires. Samples are characterized under I/V tests: a linear increasing voltage (V) from 0 to 10 Volts is applied and the corresponding current intensity (I), which flows between the electrodes, is measured. According to the Ohm law, the electrical resistance is defined as the ratio between the applied voltage and the measured current $(R=V / I)$. The voltage range 
considered is higher than the range in which this kind of device normally work, but it allows to investigate deeply the material behavior.

The nanocomposite shows a linear behavior: as voltage increases, an in increase of current intensity is recorded as shown in Figure 1 . The electrical resistance is defined as the slope of the I/V curve.

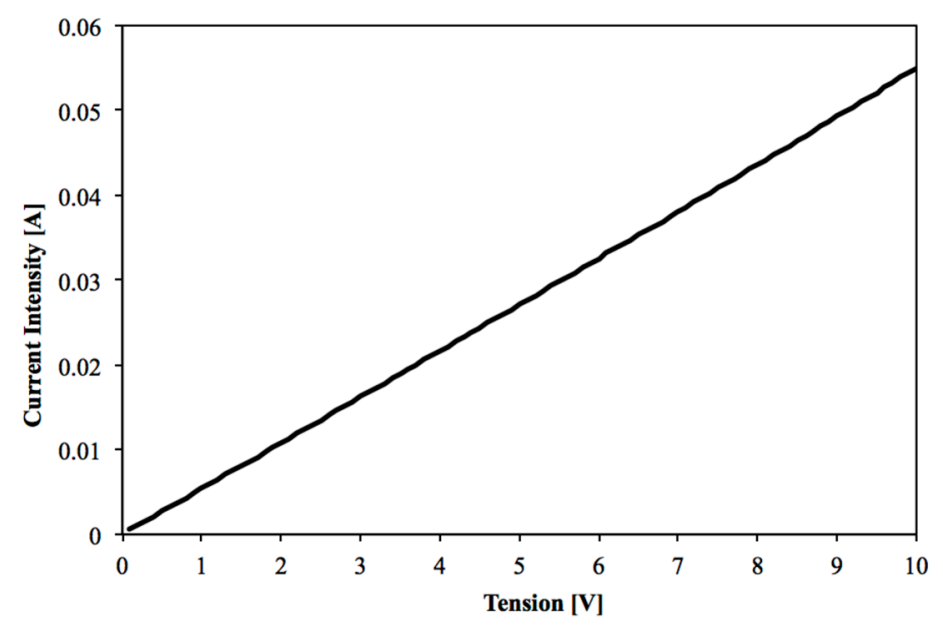

Figure 1. I/V test in stress-free condition.

The resistivity (@) of the material is calculated from the electrical resistance:

$$
\mathrm{R}=\mathrm{Ql} / \mathrm{A}
$$

(where 1 is the distance between the two electrodes, $\mathrm{A}$ is the section area of the nanocomposite) and its trend is pseudo-linear. Very slight resistivity variations between different samples form the same film, are recorded. These variations are directly related to the distribution of the MWNTs into the polymer matrix which is not completely uniform. It is worth noting that the calculated resistivity is quite low if compared with the resistivity of a film made with a 7\% MWNTs weight fraction. In the latter case, in fact, the average resistivity is $0.10 \Omega \mathrm{m}$ [7] against an average $0.004 \Omega \mathrm{m}$ of the here presented MWNT-PI nanocomposite. This behavior depends on the amount of carbon nanotubes dispersed in the polymer matrix: conductivity or resistivity of a given nanocomposite made of carbon nanotubes embedded in an insulating polymer matrix, is dictated by the number of points of contact between the nanotubes and the distance between the neighboring ones.

The electromechanical (piezoresistive) response of the MWNTs-PI nanocomposite and its capability to sense strain, is investigated with an unconventional but meaningful approach. In particular, the samples are characterized by hand stretching/unstretching the material in a cyclic manner while simultaneously monitoring its electrical response. In order to measure the electrical resistance, a constant voltage of 5 Volts is applied. Figure 2 shows snap-shots of a stretching cycle while the sample is being folded around a finger. The material flexibility is such that the film can easily be adapted to the three-dimensional shape. It is also found that, as the material is stretched, there is a corresponding electrical resistance increase. In particular, Figure 3 highlights that the electrical resistance, increases instantly, reaching a peak, when the material is deformed. As soon as the deformation is removed, the electrical resistance returns to the initial stress-free value. 

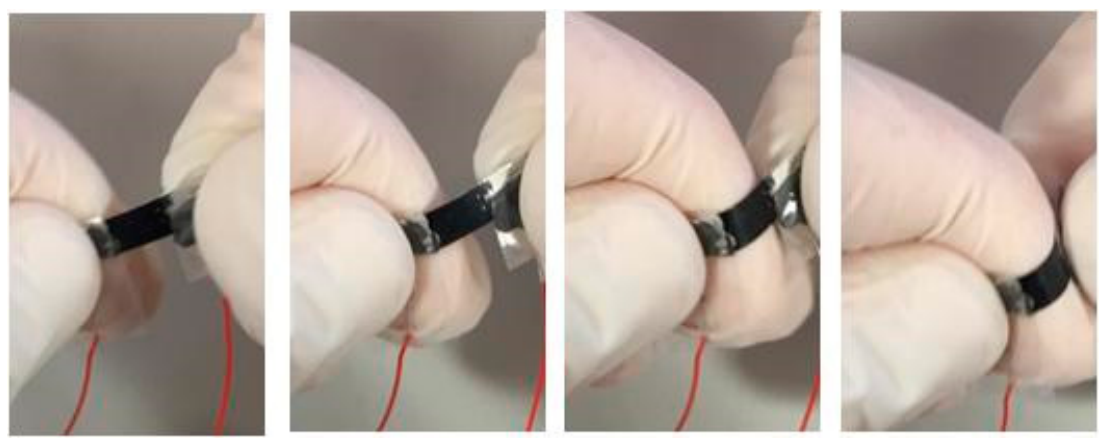

Figure 2. Snap-shots of the nanocomposite during hand-stretching and simultaneous electrical monitoring through the bonded wires.

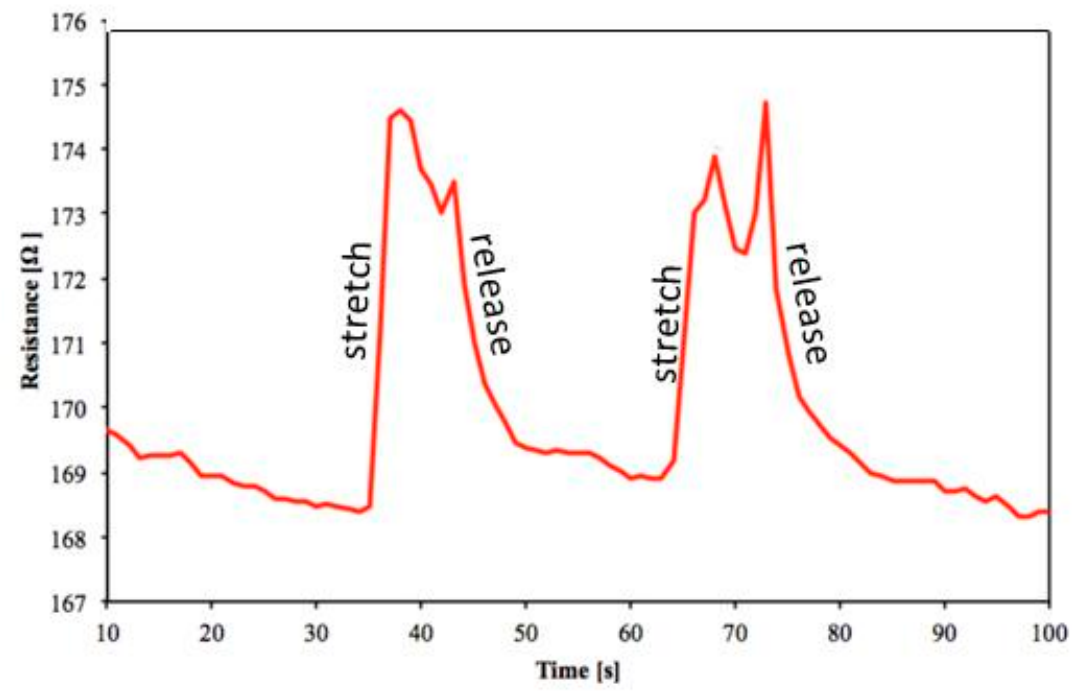

Figure 3. Electrical resistance changes when the nanocomposite is stretched.

The strain sensing capability of these nanocomposites is mainly due to the piezoresistive properties of the conductive paths formed by the carbon nanotubes embedded in the polymer matrix. When the strain increases, there is a change of the conductive paths configuration at the nanoscale, thus there is a change of the overall material conductivity. This behavior can be explained by noting that, in the material, to be electrically conductive, the CNTs must be either in direct contact or in proximity, to form a conductive network $[8,9]$. When a mechanical strain occurs, there is a loss of contact between the carbon nanotubes and an increase in distance between the neighboring CNTs. The consequence is an overall electrical resistance increase in the material.

\section{Conclusions}

In this work a MWNTs-PI nanocomposite film is presented and proposed for strain sensing applications. The I/V tests highlight a linear trend of the current vs voltage, while a linear increasing voltage (from 5 to 10 Volts) is applied. The electrical characterization also shows a two orders of magnitude decrease in material resistivity if compared with previous studies.

The piezoresistive behavior of the MWNTs-PI nanocomposite showed promising results: when the samples are stretched, instantaneous electrical resistance changes are observed. When the deformation is removed, the resistance returns to the initial value in stress-free condition.

This study highlight the large sensitivity of the MWNTs-PI nanocomposite which can be considered a promising material to realize a new kind of distributed monitoring system to monitor large structural areas. Moreover, the flexibility of the PI makes these sensors capable to adapt perfectly to the structures shapes. This is important to realize spatial and continuous monitoring systems. 
Author Contributions: G.L. conceived and designed the experiments; M.K. fabricated the samples; E.M. performed the experiments and analyzed the data; G.L. and E.M. wrote the paper.

Acknowledgments: The research leading to these results was supported by the European Research Council under the European Union's Seventh Framework Program (FP/2007-2013)/ERC Grant Agreement n. 308261.

\section{References}

1. Kanoun, O.; Müller, C.; Benchirouf, A.; Sanli, A.; Dinh, T.N.; Al-Hamry, A.; Bui, L.; Gerlach, C.; Bouhamed, A. Flexible Carbon Nanotube Films for High Performance Strain. Sensors 2014, 14, 10042-10071; doi:10.3390/s140610042.

2. Park, J.M.; Kim, D.S.; Lee, J.R.; Kim, T.W. Nondestructive damage sensitivity and reinforcing effect of carbon nanotube/epoxy composites using electro-micromechanical technique. Mater. Sci. Eng. C 2003, 23, 971-975.

3. Kang, I.; Schulz, M.J.; Kim, J.H.; Shanov, V.; Shi, D. A carbon nanotube strain sensor for structural health monitoring. Smart Mater. Struct. 2006, 15, 737-748; doi:10.1088/0964-1726/15/3/009.

4. Li, X.; Wang, W.; Liao, K.; Hu, C.; Huang, Z.; Feng, Q. Piezoresistive effect in carbon nanotube films. Chin. Sci. Bull. 2003, 48, 125-127.

5. Bu, L.; Steiz, J.; Kanoun, O. Influence of processing parameters on properties of strain sensors based on carbon nanotube films. In Proceedings of the 7th International Multi-Conference on Systems, Signals and Devices-Sensors, Circuits \& Instrumentation Systems, Amman, Jordanien, 27-30 June 2010.

6. Vemuru, S.M.; Wahi, R.; Nagarajaiah, S.; Ajayan, P.M. Strain sensing using a multiwalled carbon nanotube film. J. Strain Anal. Eng. Des. 2009, 44, 555-562.

7. Magnafico, E.; Casalotti, A.; Karimzadeh, M.; Chinnam, K.C.; Lanzara, G. Strain sensing with CNT Nanocomposites: Staic, cyclic and dynamic electromechanical material characterization. In Proceedings of the Techconnect World Innovation Conference \& Expo 20th Anniversary, Anaheim, CA, USA, 13-16 May 2018.

8. Sheng, P.; Sichel, E.K.; Gittleman, J.L. Fluctuation-induced tunneling conduction in carbonpolyvinylchloride composites. Phys. Rev. Lett. 1978, 40, 1978.

9. Simmons, J.G. Generalized formula for the electric tunnel effect between similar electrodes separated by a thin insulating film. J. Appl. Phys. 1963, 34, 1793-1803.

(C) 2018 by the authors. Licensee MDPI, Basel, Switzerland. This article is an open access article distributed under the terms and conditions of the Creative Commons Attribution (CC BY) license (http://creativecommons.org/licenses/by/4.0/). 